\section{Small Signal Analysis of the LCC-Type Parallel Resonant Converter}

ISSA BATARSEH, Senior Member, IEEE

\section{MEGALEMOS}

University of Central Florida

M. SZNAIER, Member, IEEE

Pennsylvania State University

In this paper, the small signal analysis of the LCC-type parallel resonant converter (LCC-PRC) operating in the continuous conduction mode is given. This analysis is based on both the state-plane diagram, which has been successfully used to obtain the steady state response for resonant converters, and the Taylor series expansion. Applying perturbation directly to the steady state trajectory, a discrete small signal model for the converter can be derived in terms of the input voltage, switching frequency, and the converter state variables. Based on this analysis, closed-loop form solutions for the input-to-output and control-to-output transfer functions are derived. It is shown that the theoretical and computer simulation results are in full agreement.

Manuscript received February 7, 1994; revised January 23, 1995.

IEEE Log No. T-AES/32/2/03451.

Authors' addresses: I. Batarseh and C. Megalemos, Dept. of Electrical and Computer Engineering, University of Central Florida, Orlando, FL 32816, email: batarseh@pegasus.cc.ucf.edu; M. Sznaier, Electrical Engineering Department, Pennsylvania State University, University Park, PA 16802.

$\overline{0018-9251 / 96 / \$ 10.00 ~(C) ~} 1996$ IEEE

\section{INTRODUCTION}

To achieve the desired system stability, the open-loop control-to-output frequency response is used to analytically design the transfer function of the control circuit. Furthermore, the characterization of the closed-loop small signal performance due to the line voltage is necessary to predetermine the open-loop line-to-output frequency response. Depending on the complexity of the converter topology and circuit component models used for the converter power stage, these frequency responses may be obtained experimentally, numerically, or analytically. Experimental measurements of the converter frequency responses become necessary when the converter power stage is known as a black box. On the other hand, if the circuit topology as well as the control technique of the converter power stage are known, then the power stage responses can be simulated numerically, thus avoiding the costly construction of the converter power stage. However, due to highly intensive computations, this method is time consuming. Hence, it is worthwhile to derive the analytical solutions so that excessive time and expense in the experimental approach can be avoided. It is more efficient to utilize analytical solutions for the power stage responses in the systematic design of a control circuit.

To avoid costly construction and debugging of converter prototypes based on the trial and error approach, analytical methods to obtain the small signal frequency responses for dc-to-dc converters have been developed [1-24]. The analytical small signal frequency responses help a circuit designer choose a proper compensation circuit or debug the design of the converter power stage from its steady state characteristics so that the small signal frequency responses of the revised converter and the selected compensation circuit are compatible for attaining the desired system stability. For many years, published work in this area has been focused on the pulsewidth modulation (PWM) converters [1-12]. In recent years, however, the small signal analysis of dc-to-dc resonant converters have been investigated by many researchers in this field [12-24]. Thus far, the analysis to achieve the small signal behavior is limited to second-order series and parallel resonant converters (PRCs) [13-22], with little work done on high-order resonant converters [23, 24]. This analysis is complicated in its mathematics and obscure in its physical insight into the converter operation due to a stringent time-domain analysis. An alternative analytical method is needed to easily visualize the small signal behavior around the converter steady state trajectory and to allow further generalization to a larger class of resonant converters.

The steady state analyses for numerous resonant converter topologies have been thoroughly investigated in the literature. The steady state responses of these 
converters are known and in most cases have been represented by closed trajectories in the state-plane diagram [19, 21, 28-31]. Since the state-plane diagrams of resonant converters in general consist of only a few well defined simple geometric curves in the state-plane, it is possible to develop a generalized computer program from which the frequency responses of a resonant converter can be derived once its state-plane diagram is specified. Hence, this technique can be generalized to any resonant converter topology once its state-plane trajectory is known. Moreover, by using the state-plane diagram approach, more physical insight into the converter dynamic behavior can be obtained. This is because the perturbation is done graphically on the state-plane diagram with the actual displacement of the converter state variables, as well as input and control parameters, being observed.

The analysis method was first used to achieve the discrete small signal models of the conventional series resonant converter (SRC) and PRC under variable switching frequency control in [19] and [23] respectively. In this analysis technique, the initial perturbations on the converter state variables, the control and source inputs, were made on the state-plane diagram to produce the perturbed state trajectories. Then, using the geometrical relation between the steady state and the perturbed trajectories and applying the superposition theorem, the perturbation response can be written as a linear combination of those initial perturbations. Observing the differences between the steady-state and the perturbed trajectories that are constructed on the same state-plane, we can attain better understanding of the converter small signal behavior. The discrete small signal models of these resonant converters are developed without the time-averaging of the output waveform. When the converter operates in the continuous conduction mode (CCM), the discrete small signal model can correctly predict small signal frequency response because the same set of the perturbed states exists at any given time and these perturbed states possess their continuities throughout the converter operation. This technique is applied to obtain the frequency response for the well-known LCC-type PRC. Its steady state analysis has been thoroughly analyzed in the literature [25-28]. Once the small signal model of the converter is obtained, then its closed-loop compensation can be properly designed and simulated before actually building the converter.

The steady state analysis and the derivation of the state-plane diagram for the LCC-type PRC is briefly discussed in Section II. Section III provides the mathematical development of the small signal analysis. Using Taylor series expansion coupled with the symmetry in steady state state-plane response, we derive the line-to-output and the control-to-output transfer functions. Consequently, the frequency responses can be computed from these functions.

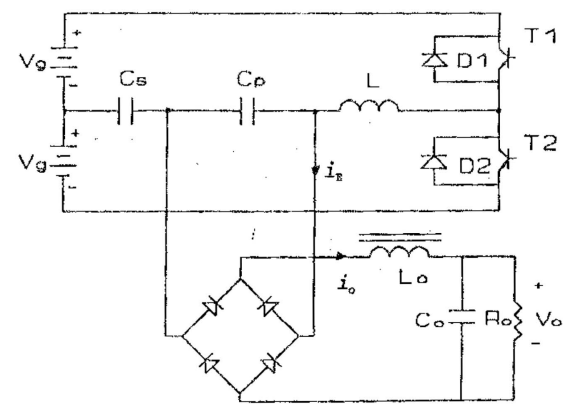

Fig. 1. Simplified circuit of LCC-type PRC.

Since it is always possible to decompose any order dc-to-dc resonant converters into many decoupled two-dimensional state-plane by using state-variable transformation technique, the small signal analysis presented here is based on two-dimensional state-plane diagram [29]. By using the state-plane diagram approach, more physical insight into the converter dynamic behavior can be obtained. This is because the perturbation is done graphically on the state-plane diagram with the actual displacements of the converter state variables and input and control parameters observed. To verify our theoretical work, computer simulation results are presented in Section IV and are compared to the theoretical ones.

\section{STEADY STATE ANALYSIS}

In order to analytically obtain the small signal response of a resonant converter, it is necessary that its dc operating conditions be known. Using this steady state trajectory, a geometrical approach can be used to derive the small signal frequency response model of the converter. In this section, steady state analysis that is based on the state-plane diagram will be briefly discussed. The objective here is to express the steady state parameters of the converter in terms of the initial switching points of the state-plane trajectory. Once such expressions are obtained, then perturbation signals on these state-variable parameters are injected directly into the state-plane diagram.

The idealized circuit of the LCC-type PRC (PRC-LCC) is shown in Fig. 1. The detailed steady state analysis of the converter can be found in [26-29]. Throughout the analysis, it is assumed that the resonant circuit is lossless and the switching devices and diodes are ideal. When the converter operates under CCM with $50 \%$ duty ratio over one switching period $T_{s}$, it can be shown that the effect of switching of transistor/diode pairs can be represented by an equivalent square wave voltage source $v_{s}(t)$ with magnitudes $+V_{g}$ and $-V_{g}$. Moreover, assuming very large $L_{0}$, the input current to the full-bridge rectifier, $i_{E}(t)$, may be presented by a dependent current source of magnitudes $+I_{0}$ and $-I_{0}$, depending on whether 


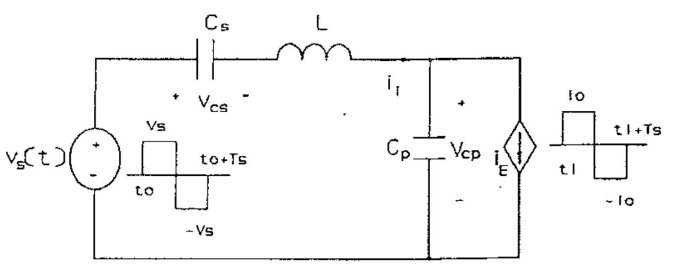

Fig. 2. Equivalent model of Fig. 1.

$v_{c p}(t)$ is greater or less than zero, respectively. Hence, the resultant equivalent circuit model is shown in Fig. 2 from which the following differential equations are obtained,

$$
\begin{aligned}
\frac{d i_{l}(t)}{d t} & =\frac{1}{L}\left[v_{s}(t)-v_{c s}(t)-v_{c p}(t)\right] \\
\frac{d v_{c p}(t)}{d t} & =\frac{1}{C_{p}}\left[i_{l}(t)-i_{E}(t)\right] \\
\frac{d v_{c s}(t)}{d t} & =\frac{1}{C_{s}}(t) .
\end{aligned}
$$

Defining a new state variable, $v_{c}(t)=v_{c p}(t)+v_{c s}(t)$, then (1)-(3) can be rewritten as follows,

$$
\begin{aligned}
\frac{d i_{l}}{d t} & =\frac{1}{L}\left[v_{s}(t)-v_{c}(t)\right] \\
\frac{d v_{c}}{d t} & =\frac{1}{C_{T}}\left[i_{l}(t)-\frac{i_{E}(t)}{\left(1+C_{p} / C_{s}\right)}\right]
\end{aligned}
$$

where $C_{T}$ is the total capacitance given by

$$
\begin{aligned}
\frac{1}{C_{T}} & =\frac{1}{C_{p}}+\frac{1}{C_{s}} \\
\frac{d v_{c}}{d i_{l}} & =\frac{L}{C_{T}} \frac{i_{l}-\frac{i_{E}}{\left(1+C_{p} / C_{s}\right)}}{v_{s}-v_{c}} .
\end{aligned}
$$

From (4) and (5), the state-plane equation in terms of $v_{c}(t)$ and $i_{1}(t)$ is given by, (6) can be expressed in terms of the normalized state-plane equation in the following form,

$$
\frac{d v_{n c}(t)}{d i_{n l}(t)}=\frac{i_{n l}(t)-i_{n E}^{\prime}(t)}{v_{n s}(t)-v_{n c}(t)}
$$

where

$$
\begin{aligned}
& v_{n c}(t)=v_{c}(t) / V_{g} \quad v_{n s}(t)=v_{s}(t) / V_{g} \\
& i_{n l}(t)=Z_{0} i_{l}(t) / V_{g} \quad i_{n E}(t)=Z_{0} i_{E}(t) / V_{g} \\
& i_{n E}^{\prime}(t)=\left[1-\left(\frac{\omega_{0 s}}{\omega_{0}}\right)^{2}\right] i_{n E}(t)
\end{aligned}
$$

and the characteristic impedance $Z_{0}$, and resonant frequencies $\omega_{0}$ and $\omega_{0 s}$ are given by

$$
Z_{0}=\sqrt{\frac{L}{C_{T}}}, \quad \omega_{0}=\frac{1}{\sqrt{L C_{T}}} \quad \text { and } \quad \omega_{0 s}=\frac{1}{\sqrt{L C_{s}}} .
$$

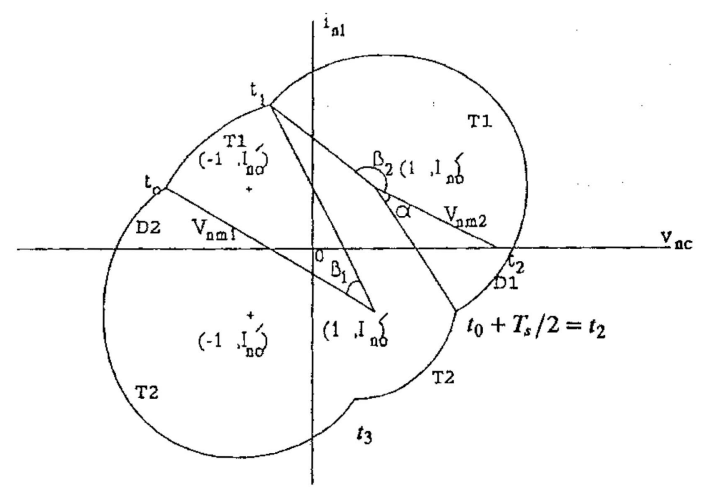

Fig. 3. Typical state-plane trajectory for LCC-PRC.

It can be shown that the solution of (7) consists of four circular arcs in the $i_{n l}-v_{n c}$ state-plane with each singular point $\left[v_{n s}, i_{n E}^{\prime}\right]$ corresponds to one of the following intervals,

$$
\left[v_{n s}, i_{n E}^{\prime}\right]= \begin{cases}\left(+V_{n g},-I_{n 0}^{\prime}\right) & t_{0} \leq t<t_{1} \\ \left(+V_{n g},+I_{n 0}^{\prime}\right) & t_{1} \leq t<t_{0}+T_{s} / 2=t_{2} \\ \left(-V_{n g},+I_{n 0}^{\prime}\right) & t_{2} \leq t<t_{3} \\ \left(-V_{n g},-I_{n 0}^{\prime}\right) & t_{3} \leq t<t_{0}+T_{s}\end{cases}
$$

where $V_{n g}=v_{g} / V_{g}$. In steady state, $V_{n g}=1$.

Typical $i_{n l}-v_{n c}$ state-plane diagram is illustrated in Fig. 3. Detailed derivation of this figure is given in [28]. In this figure, $\beta_{1}$ is the angular displacement of the trajectory when $T_{1}$ is turned on with $v_{c p}<0, \beta_{2}$ is the angular displacement of the trajectory when $T_{1}$ is conducting with $v_{c p}>0$, and $\alpha$ is the angular displacement when $D_{1}$ is conducting. These angles are related to the switching and resonant frequencies by,

$$
\gamma=\beta_{1}+\beta_{2}+\alpha=\frac{\omega_{0} T_{s}}{2}=\frac{f_{0}}{f_{s}} \pi=\frac{\pi}{f_{n s}}
$$

where $f_{s}$ and $f_{0}$ are the switching and resonant frequencies, respectively, and the normalized frequency is defined by $f_{n s}=f_{s} / f_{0}$ with $f_{0}=\omega_{0} / 2 \pi$. The solution of (7) consists of four circular arcs in the $v_{n c}-i_{n l}$ state-plane given by

$$
\left(v_{n C}-v_{n s}\right)^{2}+\left(i_{n L}-i_{n E}\right)^{2}=V_{n m i}^{2}
$$

where $i=1,2,3$, and 4 and $V_{n m i}$ are the radii which are given by

$$
V_{n m i}=\left\{\begin{array}{ll}
V_{n m 1} & \text { for } t_{0} \leq t<t_{1} \quad \text { or } t_{0}+T_{s} / 2 \leq t_{3} \\
V_{n m 2} & \text { for } t_{1} \leq t<t_{0}+T_{s} / 2 \text { or } t_{3} \leq t<t_{0}+T_{s}
\end{array} .\right.
$$

From the state-plane diagram, it can be shown that

$$
\begin{aligned}
& V_{n m 1}=\sqrt{\left(V_{n g}-v_{n C}\left(t_{0}\right)\right)^{2}+\left(i_{n L}\left(t_{0}\right)+I_{n 0}\right)^{2}} \\
& V_{n m 2}=\sqrt{\left(i_{n L}\left(t_{1}\right)-I_{n 0}\right)^{2}+V_{n g}^{2}} .
\end{aligned}
$$


The dc output voltage $V_{0}$ is obtained by taking the average value of the resonant capacitor voltage, $v_{c p}$, to yield

$$
V_{0}=\frac{1}{\gamma}\left[1-\left(\frac{\omega_{0 s}}{\omega_{0}}\right)^{2}\right]\left[\left(\gamma-2 \beta_{1}\right) V_{g}+2 Z_{0} i_{l}\left(t_{1}\right)\right]
$$

where

$$
\beta_{1}=\omega_{0}\left(t_{1}-t_{0}\right) .
$$

The converter gain is obtained by normalizing (14) to give

$$
M=\frac{V_{0}}{V_{g}}=\frac{1}{\gamma}\left[1-\left(\frac{\omega_{0 s}}{\omega_{0}}\right)^{2}\right]\left[\left(\gamma-2 \beta_{1}\right)+2 i_{n l}\left(t_{1}\right)\right] .
$$

Since the steady state analysis shown here is only for the PRC-LCC operating in the natural commutation mode, $i_{n l}\left(t_{0}\right)$ must be positive and it can be expressed as

$$
i_{n l}\left(t_{0}\right)=\sqrt{I_{n 0}^{2}+\left(\frac{1}{I_{n 0}^{2}}-1\right) v_{n C}^{2}\left(t_{0}\right)}-I_{n 0} .
$$

For the converter design, it is more convenient to express the output voltage and converter gain in terms of the initial switching points $v_{n c}\left(t_{0}\right)$ or $i_{n l}\left(t_{0}\right)$ instead of $i_{n l}\left(t_{1}\right)$. From the state-plane diagram given in Fig. 3 , it can be shown that the following relation can be obtained,

$$
i_{n l}\left(t_{1}\right)=-\frac{v_{n c}\left(t_{0}\right)}{I_{n 0}^{\prime}} .
$$

Substituting (17) into (15), the converter gain becomes

$$
M=\frac{1}{\gamma}\left[1-\left(\frac{\omega_{0 s}}{\omega_{0}}\right)^{2}\right]\left[\left(\gamma-2 \beta_{1}\right)-\frac{2 v_{n c}\left(t_{0}\right)}{I_{n 0}^{\prime}}\right] .
$$

Finally, we need to express the switching angles $\beta_{1}$, $\beta_{2}, \alpha$, and $\gamma$ in terms of the initial switching point $\left[v_{n c}\left(t_{0}\right), i_{n l}\left(t_{0}\right)\right]$. This can be accomplished from the state-plane diagram given in Fig. 3 to obtain the following relations,

$$
\begin{gathered}
\beta_{1}=\tan ^{-1} \frac{i_{n l}\left(t_{1}\right)+I_{n 0}^{\prime}}{1-v_{n c}\left(t_{1}\right)}-\tan ^{-1} \frac{i_{n l}\left(t_{0}\right)+I_{n 0}^{\prime}}{1-v_{n c}\left(t_{0}\right)} \\
\beta_{2}+\alpha=\frac{3 \pi}{2}-\tan ^{-1} \frac{i_{n l}\left(t_{1}\right)-I_{n 0}^{\prime}}{1-v_{n c}\left(t_{1}\right)}-\tan ^{-1} \frac{i_{n l}\left(t_{0}\right)+I_{n 0}^{\prime}}{1+v_{n c}\left(t_{0}\right)} .
\end{gathered}
$$

Using (9), (19), and (20), the following functional expression for $\gamma$ can be obtained as follows,

$$
\gamma=\gamma\left[v_{n c}\left(t_{0}\right), i_{n l}\left(t_{0}\right), I_{n 0}^{\prime}, \omega_{0} / \omega_{0 s}\right] .
$$

For the converter design, given $\omega_{0} / \omega_{0 s}$, we may calculate $I_{n 0}^{\prime}, M$, and $\gamma$ once the initial switching point $\left[v_{n c}\left(t_{0}\right), i_{n l}\left(t_{0}\right)\right]$ is known. In the following section, based on the steady state equations derived in Section II, we derive the small signal model by injecting perturbation to the state-variables of the state-plane.

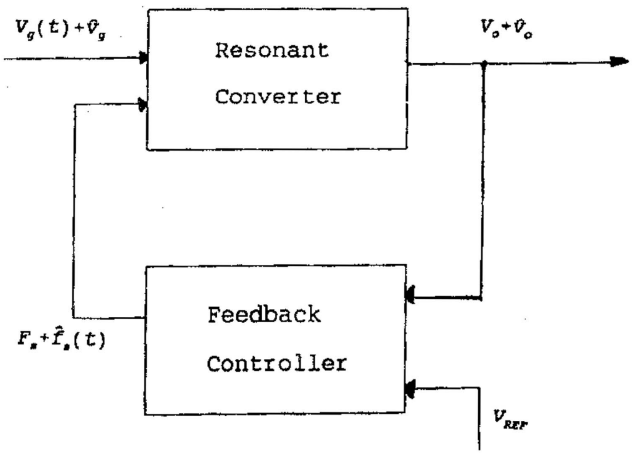

Fig. 4. Converter block diagram under line and control signals perturbations.

\section{SMALL SIGNAL ANALYSIS}

As shown in Section II, when the converter response is in steady state, the trajectory of its state variables forms a unique closed contour for every switching period with stable dc values. However, the converter trajectory can deviate from this unique contour due to disturbances on the line and control inputs. In such a case, the trajectory becomes a perturbed trajectory. Under small signal perturbation, the excursion of the perturbed trajectory from the steady state trajectory can be physically observed on the state-plane diagram [22, 23]. Physically, the small signal excursions from the steady state trajectory can be observed as the perturbed trajectory periodically shrinks and expands around the steady state trajectory at the frequency of perturbation. Major sources of perturbations are due to the input and frequency variations. Fig. 4 shows a simplified block diagram for a closed-loop converter system under line voltage $v_{g}$, and control signal (switching frequency) $f_{s}$, perturbations. Based on this model, we derive closed-form solutions for the line-to-output transfer function $H_{1}(s)$ and control-to-output transfer function $\mathrm{H}_{2}(\mathrm{~s})$ which are defined as follows,

$$
\begin{aligned}
& H_{1}(s)=\frac{\hat{v}_{0}}{\hat{v}_{g}} \\
& H_{2}(s)=\frac{\hat{v}_{0}}{\hat{f}_{s}} .
\end{aligned}
$$

In this section, based on the state-plane diagram and the generalized analysis presented in [21], the small signal analysis for the PRC-LCC when operated in the CCM is derived. By introducing perturbations to the converter state variables, controlled switching frequency, and the input voltage at the beginning of a half switching period, we can obtain the perturbed response at the beginning of the next half switching period by using Taylor series expansion. Based on this technique, a closed form solution for $H_{1}(s)$ and $H_{2}(s)$ is derived from which we obtain the frequency response. 


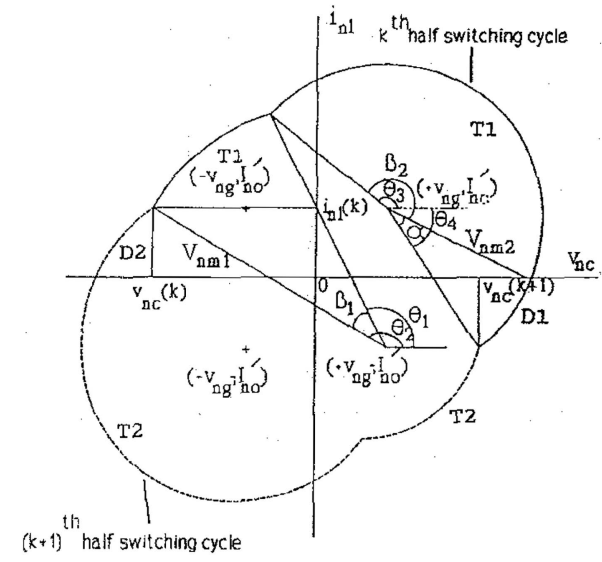

Fig. 5. Perturbed steady state trajectory of Fig. 3.

Under small signal perturbations, we define $k$ th and $(k+1)$ th as trajectories in the first and second half switching cycles of the state-plane diagram of Fig. 4, respectively, as shown in Fig. 5. Using this figure, the state-variables at the end of the half switching cycle can be related to the state-variables at the beginning of the half switching cycle, the normalized voltage $V_{n g}$, and the normalized switching frequency $f_{n s}$. The beginning and the final normalized state variables at the $k$ th half switching cycle are defined as follows:

\begin{tabular}{|c|c|}
\hline${ }_{l}(k)$ & $\begin{array}{l}\text { esonant current at the beginning of } \\
\text { th half switching cycle, }\end{array}$ \\
\hline$v_{n c}(k)$ & $\begin{array}{l}\text { the beginning of } t \\
\text { cycle, }\end{array}$ \\
\hline$f_{n s}(k)$ & ency for the $k$ th half \\
\hline$I_{n 0}(k)$ & $\begin{array}{l}\text { output current for the } k \text { th half } \\
\text { switching cycle, }\end{array}$ \\
\hline$i_{n l}(k+1)$ & $\begin{array}{l}\text { resonant current at the end of } k \text { th half } \\
\text { switching cycle, }\end{array}$ \\
\hline$v_{n c}(k+1)$ & $\begin{array}{l}\text { resonant voltage at the end of } k \text { th half } \\
\text { switching cycle, }\end{array}$ \\
\hline$I_{n 0}(k+$ & $\begin{array}{l}\text { output current for the }(k+1) \text { th half } \\
\text { switching cycle. }\end{array}$ \\
\hline
\end{tabular}

In this analysis, $\hat{\mathbf{P}}(k)$ and $\hat{\mathbf{P}}(k+1)$ are defined as the perturbation vectors at the steady state turn-on instants of transistors $T_{1}$ and $T_{2}$, respectively, which are defined as follows:

$$
\begin{aligned}
\hat{\mathbf{P}}(k) & =\left[\hat{\imath}_{n l}(k) \hat{v}_{n c}(k) \hat{I}_{n 0}(k)\right]^{T} \\
\hat{\mathbf{P}}(k+1) & =\left[\hat{\imath}_{n l}(k+1) \hat{v}_{n c}(k+1) \hat{I}_{n 0}(k+1)\right]^{T} .
\end{aligned}
$$

The perturbation vectors given in (23) and (24) represent the perturbation responses at the end of the second half and first half of the switching cycle, respectively. In another words, the perturbation responses at the end of the on-time interval of transistor $T_{1}, \hat{\imath}_{n L}(k+1), \hat{v}_{n C}(k+1)$ and $\hat{\imath}_{n 0}(k)$ are defined as the perturbation vector $\hat{\mathbf{P}}(k+1)$. Hence, the perturbation states $\hat{i}_{n L}(k)$ and $\hat{v}_{n C}(k)$ can be considered as the initial state perturbations at the beginning of the on-time interval of transistor $T_{1}$. The perturbation states $\hat{i}_{n L}(t)$ and $\hat{v}_{n C}(t)$ are continuous time functions within the given half switching period. However, only $\hat{\imath}_{n L}(k+1)$ and $\hat{v}_{n C}(k+1)$ are used as the updated perturbations for the following half switching period as shown in Fig. 5. On the other hand, the perturbations due to input voltage and output current, $\hat{v}_{n g}(t)$ and $\hat{\imath}_{n 0}(t)$, are considered to be constant within each half switching period and are updated only at the end of the half switching period. This is valid under the assumption that the input and output filter time constants are large as compared with the operating switching period $T_{s}$. Moreover, the perturbation vector $\hat{\mathbf{P}}(k)$ is assumed to be small enough so that when applying Taylor series expansion second and higher order terms will be neglected as is shown in the next section.

Two sets of discrete state equations can be obtained geometrically; one from the trajectory in even portion of the switching period and the other in the odd half switching period. These sets of state equations can be represented as two vector-matrix discrete state equations having different coefficient matrices. Since the steady state trajectory is symmetrical about the origin, special transformation on the perturbed state variables can be applied to both sets of equations to obtain a closed-form vector-matrix state equation which is valid in any half switching period. Consequently, small signal frequency responses can be calculated from the closed-form state equation.

The perturbation responses $\hat{\imath}_{n L}(k+1)$ and $\hat{v}_{n C}(k+$ 1) are derived by using the geometry of the state-plane diagram and the application of Taylor series expansion. These solutions can then be used to obtain the perturbation response $\hat{\imath}_{n 0}(k+1)$ from the output equation which is derived from the circuit topology as shown in (25). Due to the presence of the full-bridge rectifier at the output circuit of the PRC-LCC, the output equation $i_{0}(t)$ is given by

$$
L_{0} \frac{d i_{0}}{d t}=\left|v_{c p}(t)\right|-i_{0} R_{0} .
$$

From (25) and the state-plane diagram, the discrete model for the $k$ th half switching cycle, which is located at the first half of the switching cycle, can be expressed as follows,

$$
\begin{aligned}
i_{n l}(k+1)= & I_{n 0}^{l}(k)+V_{n m 2} \sin \left(2 \pi+\theta_{4}\right) \\
v_{n c}(k+1)= & v_{n g}(k)+V_{n m 2} \cos \left(2 \pi+\theta_{4}\right) \\
I_{n 0}^{\prime}(k+1)= & I_{n 0}^{\prime}(k)+\frac{Z_{0}}{\omega_{0} L_{0}}\left[1-\left(\frac{\omega_{0 s}}{\omega_{0}}\right)^{2}\right]^{2} \\
& \times\left(\frac{\pi}{f_{n s}(k)}-2 \beta_{1}\right)-2 \frac{L}{L_{0}}\left[1-\left(\frac{\omega_{0 s}}{\omega_{0}}\right)^{2}\right]^{2} \\
& \times \frac{V_{n c}(k)}{I_{n 0}^{\prime}(k)}-\frac{\pi R_{0} I_{n 0}^{\prime}(k)}{L_{0} \omega_{0} f_{n s}(k)} .
\end{aligned}
$$


In order to find a complete discrete state model that applies for both the first and second half switching cycles, new state variables need to be defined [21] in terms of the absolute values of the converter state variables. Hence, a closed-form solution for the upper and lower switching cycles can be obtained. It is clear from the state-plane diagram shown in Fig. 5 that the absolute values of the state variables must be used as follows:

$$
\begin{aligned}
y(k) & =\left|i_{n l}(k)\right|=i_{n l}(k) \\
x(k) & =\left|V_{n c}(k)\right|=-v_{n c}(k) \\
y(k+1) & =\left|i_{n l}(k+1)\right|=-i_{n l}(k+1) \\
x(k+1) & =\left|v_{n c}(k+1)\right|=v_{n c}(k+1) .
\end{aligned}
$$

Based on these new variables, the perturbation vectors $\mathbf{P}(k)$ and $\mathbf{P}(k+1)$ may redefined as shown in (29) and (30), respectively,

$$
\begin{aligned}
\mathbf{Q}(k) & =\mathbf{P}^{\mathbf{T}}(k)\left[\begin{array}{rrr}
1 & 0 & 0 \\
0 & -1 & 0 \\
0 & 0 & 1
\end{array}\right] \\
& =\left[\begin{array}{lll}
y(k) & x(k) & I_{n 0}^{\prime}(k)
\end{array}\right] \\
\mathbf{Q}(k+1) & =\mathbf{P}^{\mathbf{T}}(k+1)\left[\begin{array}{rrr}
1 & 0 & 0 \\
0 & -1 & 0 \\
0 & 0 & 1
\end{array}\right] \\
& =\left[\begin{array}{lll}
y(k+1) & x(k+1) & I_{n 0}^{\prime}(k+1)
\end{array}\right] .
\end{aligned}
$$

By applying the Taylor series expansion into the steady state discrete model described by (26)-(28) and neglecting the higher order terms, we obtain the responses for $\hat{y}(k+1), \hat{x}(k+1)$, and $\hat{I}_{n 0}(k+1)$ as shown in (31), (32), and (33), respectively,

$$
\begin{aligned}
\hat{y}(k+1)= & \frac{\partial F_{1}}{\partial y(k)} \hat{y}(k)+\frac{\partial F_{1}}{\partial x(k)} \hat{x}(k)+\frac{\partial F_{1}}{\partial I_{n 0}^{\prime}(k)} \hat{I}_{n 0}^{\prime}(k) \\
& +\frac{\partial F_{1}}{\partial f_{n s}(k)} \hat{f}_{n s}(k)+\frac{\partial F_{1}}{\partial V_{v g}(k)} \hat{V}_{n g}(k) \\
\hat{x}(k+1)= & \frac{\partial F_{2}}{\partial y(k)} \hat{y}(k)+\frac{\partial F_{2}}{\partial x(k)} \hat{x}(k)+\frac{\partial F_{2}}{\partial I_{n 0}^{\prime}(k)} \hat{I}_{n 0}^{\prime}(k) \\
& +\frac{\partial F_{2}}{\partial f_{n s}(k)} \hat{f}_{n s}(k)+\frac{\partial F_{2}}{\partial V_{v g}(k)} \hat{V}_{n g}(k) \\
\hat{I}_{n 0}^{\prime}(k+1)= & \frac{\partial F_{3}}{\partial y(k)} \hat{y}(k)+\frac{\partial F_{3}}{\partial x(k)} \hat{x}(k)+\frac{\partial F_{3}}{\partial I_{n 0}^{\prime}(k)} \hat{I}_{n 0}^{\prime}(k) \\
& +\frac{\partial F_{3}}{\partial f_{n s}(k)} \hat{f}_{n s}(k)+\frac{\partial F_{3}}{\partial V_{v g}(k)} \hat{V}_{n g}(k) .
\end{aligned}
$$

The partial derivatives shown in (31)-(33) are derived in the Appendix. In a more compact form, (31)-(33) may be represented as follows,

$$
\mathbf{Q}(k+1)=\mathbf{A} \mathbf{Q}(k)+\mathbf{B} \hat{f}_{n s}(k)+\mathbf{C} \hat{V}_{n g}(k)
$$

where

$$
\mathbf{A}=\left[\begin{array}{lll}
a_{11} & a_{12} & a_{13} \\
a_{21} & a_{22} & a_{23} \\
a_{31} & a_{32} & a_{33}
\end{array}\right], \quad \mathbf{B}=\left[\begin{array}{l}
b_{1} \\
b_{2} \\
b_{3}
\end{array}\right], \quad \mathbf{C}=\left[\begin{array}{l}
c_{1} \\
c_{2} \\
c_{3}
\end{array}\right] .
$$

The coefficients of matrices $\mathbf{A}, \mathbf{B}$, and $\mathbf{C}$ are given in the Appendix.

To find the small signal frequency response of the converter, the discrete small signal model of (34) needs to be represented in the frequency domain using $z$-transformation as shown in (35),

$$
\mathbf{Q}(z)=(z \mathbf{I}-\mathbf{A})^{-1} \mathbf{B} \hat{f}_{n s}(z)+(z \mathbf{I}-\mathbf{A})^{-1} \mathbf{C} \hat{V}_{n g}(z) .
$$

The line-to-output transfer function in the $z$-domain can be obtained by letting $\hat{f}_{s n}(z)$ be zero in (35) to obtain

$$
H_{1}(s)=\frac{\hat{V}_{n 0}(z)}{\hat{V}_{n g}(z)}=\left[\frac{Q_{p}}{1-\left(\frac{\omega_{0 s}}{\omega_{0}}\right)^{2}}\right]\left[\begin{array}{lll}
0 & 0 & 1
\end{array}\right][z \mathbf{I}-\mathbf{A}]^{-1} \mathbf{C}
$$

where $Q_{p}$ is the quality factor defined by,

$$
\mathbf{Q}_{p}=\frac{\mathbf{R}_{0}}{\mathbf{Z}_{0}} .
$$

Similarly, the control-to-output transfer function in the $z$-domain can be obtained by setting $\hat{V}_{n g}(z)$ to zero in (35) and using normalized factor as shown in (38),

$$
H_{2}(s)=\frac{\hat{V}_{n 0}(z)}{\hat{f}_{s n}(z)}=\left[\frac{Q_{p}}{1-\left(\frac{\omega_{0 s}}{\omega_{0}}\right)^{2}}\right]\left[\begin{array}{lll}
0 & 0 & 1
\end{array}\right][z \mathbf{I}-\mathbf{A}]^{-1} \mathbf{B} .
$$

The Bode plots, both magnitude and phase, of the line-to-output transfer function $H_{1}(s)$, and the control-to-output transfer function $\mathrm{H}_{2}(s)$, based on (36) and (38), are shown in Figs. 6 and 7, respectively.

\section{SIMULATION RESULTS}

By using the Pspice simulation program, the frequency responses for both transfer functions are obtained to verify the theoretical work. Under small signal perturbations, the driving source in the equivalent circuit is amplitude modulated signal for the line-to-output response and frequency modulated signal for the control-to-output response.

\section{A. Line-to-Output Frequency Response}

The setup for the line-to-output response is simple as shown in the simulated circuit of Fig. 8. The 

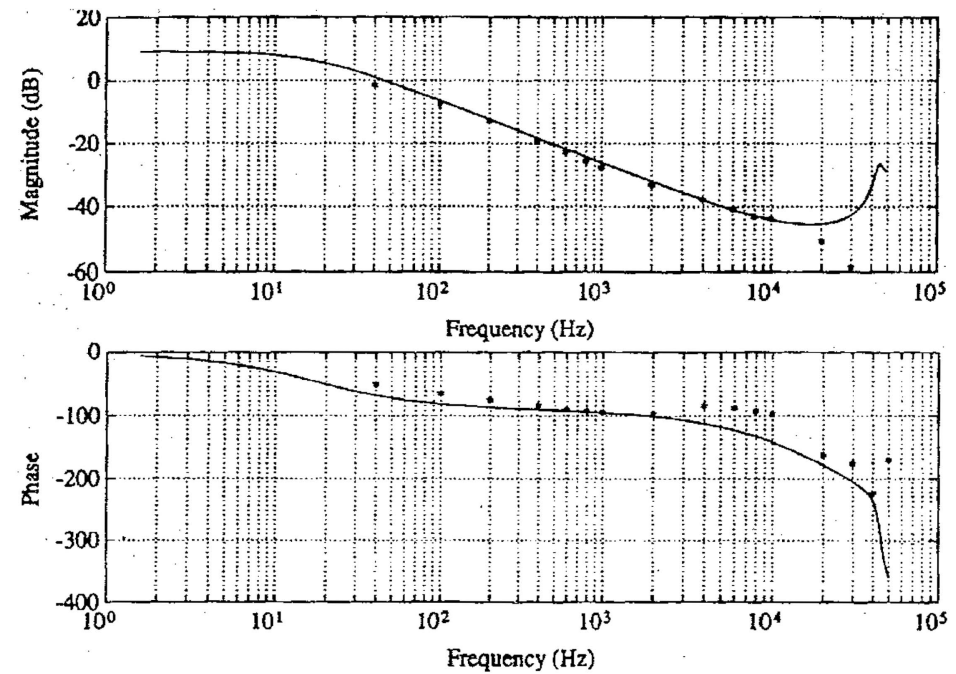

Fig. 6. Line-to-output frequency response. Theoretical results: solid line. Simulated results: broken line.
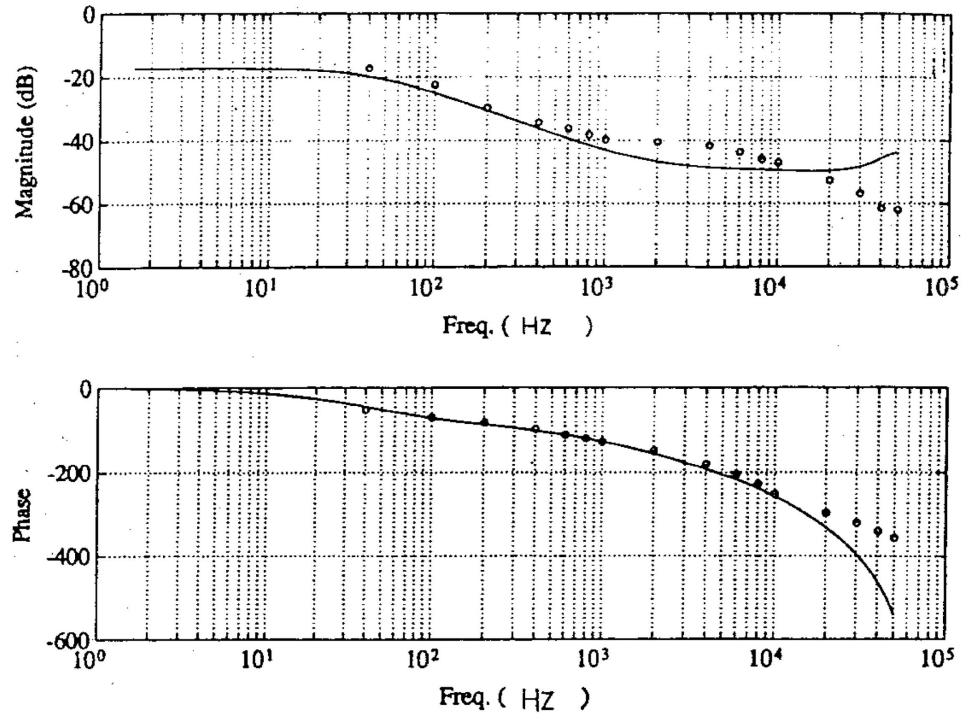

Fig. 7. Control-to-output frequency response. Theoretical results: solid line. Simulated results: broken line.

converter values are based on a design example for the following values:

$\begin{array}{ll}\text { DC input voltage } & V_{g}=10 \mathrm{~V} \\ \text { Load current } & I_{0}=5 \mathrm{~A} \\ \text { Output voltage } & V_{0}=15 \mathrm{~V} \\ \text { Switching frequency } & f_{s}=100 \mathrm{kHz} \\ \text { Capacitor ratio } & C_{p} / C_{s}=0.5\end{array}$

The converter component values are given as follows:

$$
\begin{gathered}
R_{0}=3 \Omega, \quad L_{0}=20 \mathrm{mH}, \quad L=3.1252 \mu \mathrm{H}, \\
C_{p}=1.63 \mu, \quad C_{s}=1.63 \mu .
\end{gathered}
$$

Source $v_{A}(t)$ is a $50 \%$ duty ratio square wave signal with switching frequency of $100 \mathrm{kHz}$ and amplitudes of $\pm 10 \mathrm{~V}$. Source $v_{F}(t)$ generates the small signal

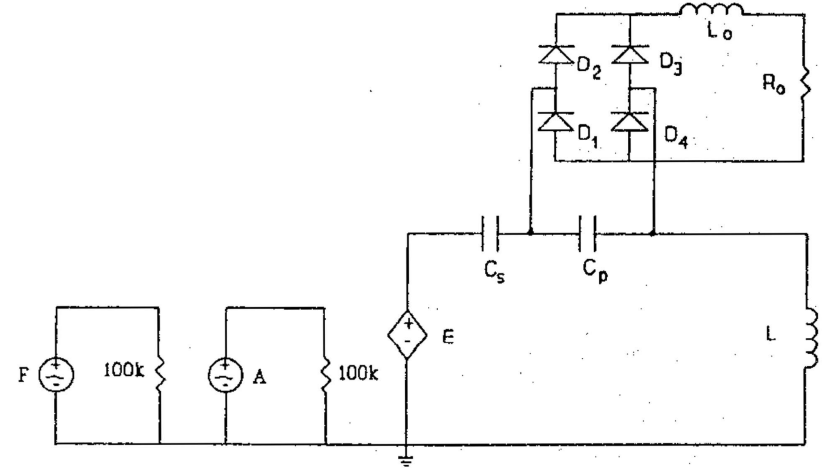

Fig. 8. Simulated circuit for line-to-output response.

perturbation with dc offset of $10 \mathrm{~V}\left(v_{F}(t)=10+\right.$ $\left.\sin \left(2 \pi f_{m} t\right)\right)$, where $f_{m}$ is the small signal frequency between $10 \mathrm{~Hz}$ and $100 \mathrm{kHz}$. Source $v_{E}(t)$ is a dependent signal which generates the square wave 

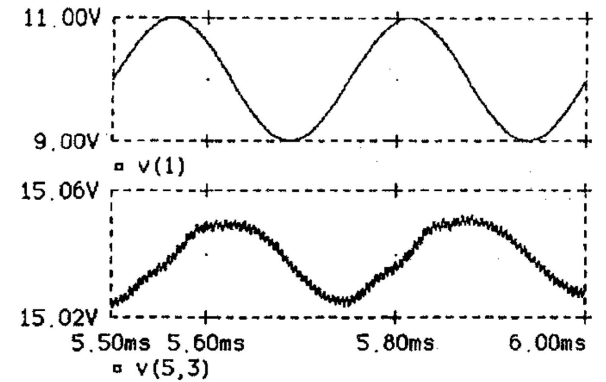

Fig. 9. Simulated waveforms for $4 \mathrm{kHz}$ perturbation signal for Fig. 8. $v(1)$ : input (reference) voltage. $v(5,3)$ : output voltage.

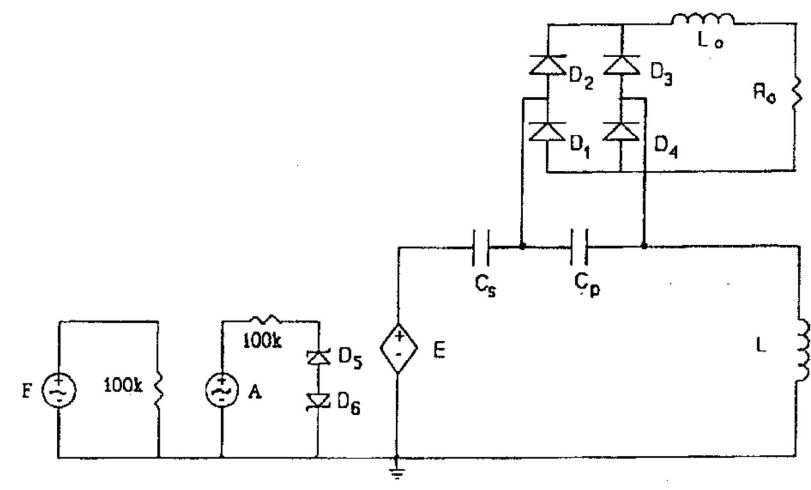

Fig. 10. Simulated circuit for control-to-output response.

amplitude modulated signal with peak amplitude of $10 \mathrm{~V}\left(v_{E}(t)=0.1 v_{F}(t) v_{A}(t)\right)$. Fig. 9 shows the output response at perturbation signal of $4 \mathrm{kHz}$. The simulation results for the line-to-output frequency response is shown in Fig. 6.

\section{B. Control-to-Output Frequency Response}

The simulated circuit for the control-to-output response is shown in Fig. 10 with the same converter components given in Fig. 9. In Fig. 10, the source $v_{A}(t)$ is frequency modulated signal, $v_{F}(t)$ is used as a small signal reference source, and $v_{E}(t)$ is a dependent source that generates the square wave single frequency modulated signal. These sources are defined as follows.

Source A: $\quad v_{A}(t)=A_{1} \sin \left[2 \pi f_{c} t+B \sin \left(2 \pi f_{m} t\right)\right.$ where

$A_{1} \quad 500 \mathrm{~V}$,

$f_{c} \quad 100 \mathrm{kHz}$ (switching frequency)

$f_{m} \quad$ Small signal frequency $\left(10 \mathrm{~Hz} f_{m}<\right.$ $100 \mathrm{kHz}$ ),

$\Delta_{f} \quad 1.0 \% f_{c}$ (switching frequency deviation)

$B \quad$ Modulation index $\left(B=\Delta f / f_{m}\right)$.

Source $F: \quad v_{F}(t)=A_{2} \cos \left(2 \pi f_{m} t\right)$ where

$A_{2} \quad 0.05 \mathrm{~V}$ (small signal amplitude)

$f_{m}$ Perturbation frequency $\left(10 \mathrm{~Hz}<f_{m}<\right.$ $100 \mathrm{kHz})$.
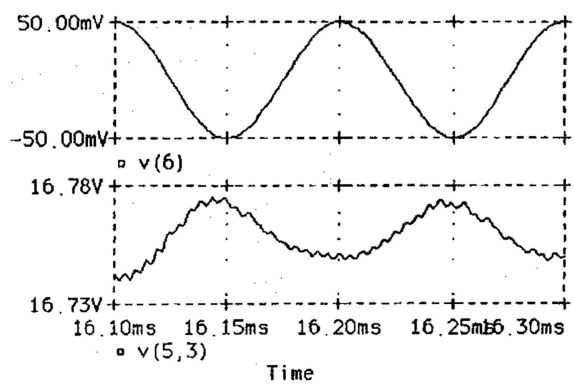

Fig. 11. Simulated waveforms for $10 \mathrm{kHz}$ perturbation signal for Fig. 10. $v(6)$ : input (reference) voltage. $v(5,3)$ : output voltage.

Source E: $\quad v_{E}(t)=V_{z}(t)$ where the zener voltages of the diodes are set to $10 \mathrm{~V}$.

Fig. 11 shows the output response due to the control frequency perturbation at $10 \mathrm{kHz}$. The simulation results are also plotted in Fig. 7. Theses results show that the frequency responses from both the theory and simulation are in good agreement, especially at low frequencies.

\section{CONCLUSION}

The small signal analysis for the LCC-PRC operating in the CCM has been presented. The closed form solution of the small signal response can be obtained from the state-plane diagram with the application of Taylor series expansion. Two related transfer functions under line and control perturbations were derived: line-to-output and control-to-output transfer functions. Using $z$-transformation, frequency responses for these two transfer functions were derived. Finally, simulated results for the magnitude and phase responses of the transfer functions were reported and compared to verify the theoretical approach. It was shown that the simulated results are in good agreement with the theoretical results, especially at low frequencies. Since accurate knowledge of the transfer function is more critical close to the cross-over frequency (i.e., for magnitudes close to one), the relatively high error at high frequencies poses no problems.

\section{APPENDIX}

A. Derivation of Partial Derivatives for (32)

The coefficients of matrices $\mathbf{A}, \mathbf{B}$, and $\mathbf{C}$ associated with $\hat{y}(k+1)$ :

$$
\begin{aligned}
\frac{\partial F_{1}}{\partial y(k)}=a_{11}=- & {\left[\frac{\partial V_{n m 2}}{\partial y(k)} \sin \theta_{4}+V_{n m 2} \cos \theta_{4}\right.} \\
& \left.\times\left(\frac{\partial \theta_{2}}{\partial y(k)}-\frac{\partial \theta_{1}}{\partial y(k)}+\frac{\partial \theta_{3}}{\partial y(k)}\right)\right]
\end{aligned}
$$




$$
\begin{aligned}
\frac{\partial F_{1}}{\partial x(k)}=a_{12}=- & {\left[\frac{\partial V_{n m 2}}{\partial x(k)} \sin \theta_{4}+V_{n m 2} \cos \theta_{4}\right.} \\
& \left.\times\left(\frac{\partial \theta_{2}}{\partial x(k)}-\frac{\partial \theta_{1}}{\partial x(k)}+\frac{\partial \theta_{3}}{\partial x(k)}\right)\right] \\
\frac{\partial F_{1}}{\partial I_{n 0}(k)}=a_{13}=- & {\left[1+\frac{\partial V_{n m 2}}{\partial I_{n 0}^{\prime}(k)} \sin \theta_{4}+V_{n m 2} \cos \theta_{4}\right.} \\
& \left.\times\left(\frac{\partial \theta_{2}}{\partial I_{n 0}^{\prime}(k)}-\frac{\partial \theta_{1}}{\partial I_{n 0}^{\prime}(k)}+\frac{\partial \theta_{3}}{\partial I_{n 0}^{\prime}(k)}\right)\right] \\
\frac{\partial F_{1}}{\partial f_{n s}(k)}=b_{1}=- & V_{n m 2} \cos \theta_{4}\left(\frac{\pi}{F_{n s}^{2}}\right) \\
\frac{\partial F_{1}}{\partial V_{n g}(k)}=c_{1}=- & {\left[\frac{\partial V_{n m 2}}{\partial V_{n g}(k)} \sin \theta_{4}+V_{n m 2} \cos \theta_{4}\right.} \\
& \left.\times\left(\frac{\partial \theta_{2}}{\partial V_{n g}(k)}-\frac{\partial \theta_{1}}{\partial V_{n g}(k)}+\frac{\partial \theta_{3}}{\partial V_{n g}(k)}\right)\right] .
\end{aligned}
$$

B. Derivation of Partial Derivatives for (33)

The coefficients of matrices $\mathbf{A}, \mathbf{B}$, and $\mathbf{C}$ associated with $\hat{x}(k+1)$ :

$$
\begin{aligned}
\frac{\partial F_{2}}{\partial y(k)}= & a_{21}=\frac{\partial V_{n m 2}}{\partial y(k)} \cos \theta_{4} \\
& -V_{n m 2} \sin \theta_{4}\left(\frac{\partial \theta_{2}}{\partial y(k)}-\frac{\partial \theta_{1}}{\partial y(k)}+\frac{\partial \theta_{3}}{\partial y(k)}\right) \\
\frac{\partial F_{2}}{\partial x(k)}= & a_{22}=\frac{\partial V_{n m 2}}{\partial x(k)} \cos \theta_{4} \\
& -V_{n m 2} \sin \theta_{4}\left(\frac{\partial \theta_{2}}{\partial x(k)}-\frac{\partial \theta_{1}}{\partial x(k)}+\frac{\partial \theta_{3}}{\partial x(k)}\right) \\
\frac{\partial F_{2}}{\partial I_{n 0}(k)}= & a_{23}=\frac{\partial V_{n m 2}}{\partial I_{n 0}^{\prime}(k)} \cos \theta_{4} \\
& -V_{n m 2} \sin \theta_{4}\left(\frac{\partial \theta_{2}}{\partial I_{n 0}^{\prime}(k)}-\frac{\partial \theta_{1}}{\partial I_{n 0}^{\prime}(k)}+\frac{\partial \theta_{3}}{\partial I_{n 0}^{\prime}(k)}\right) \\
\frac{\partial F_{2}}{\partial f_{n s}(k)}= & b_{2}=-V_{n m 2} \sin \theta_{4}\left(\frac{\pi}{F_{n s}^{2}}\right) \\
\frac{\partial F_{2}}{\partial V_{n g}(k)}= & c_{2}=1+\frac{\partial V_{n m 2}}{\partial V_{n g}(k)} \cos \theta_{4} \\
& -V_{n m 2} \sin \theta_{4}\left(\frac{\partial \theta_{2}}{\partial V_{n g}(k)}-\frac{\partial \theta_{1}}{\partial V_{n g}(k)}+\frac{\partial \theta_{3}}{\partial V_{n g}(k)}\right) .
\end{aligned}
$$

C. Derivation of Partial Derivatives for (34)

The coefficients of matrices $\mathbf{A}, \mathbf{B}$, and $\mathbf{C}$ associated with $\hat{I}_{n 0}^{\prime}(k+1)$ :

$$
\frac{\partial F_{3}}{\partial y(k)}=a_{31}=-\frac{2 Z_{0}}{\omega_{0} L_{0}}\left[1-\left(\frac{\omega_{0 s}}{\omega_{0}}\right)^{2}\right]^{2}\left(\frac{\partial \theta_{2}}{\partial y(k)}-\frac{\partial \theta_{1}}{\partial y(k)}\right)
$$

$$
\begin{aligned}
\frac{\partial F_{3}}{\partial x(k)}= & a_{32}=-\frac{2 Z_{0}}{\omega_{0} L_{0}}\left[1-\left(\frac{\omega_{0 s}}{\omega_{0}}\right)^{2}\right]^{2}\left(\frac{\partial \theta_{2}}{\partial x(k)}-\frac{\partial \theta_{1}}{\partial x(k)}\right) \\
& +\frac{2 L_{1}}{L_{0}}\left[1-\left(\frac{\omega_{0 s}}{\omega_{0}}\right)^{2}\right]^{2} \frac{1}{I_{n 0}^{\prime}}(k) \\
\frac{\partial F_{3}}{\partial I_{n 0}^{\prime}(k)}= & a_{33}=1-\frac{2 Z_{0}}{\omega_{0} L_{0}}\left[1-\left(\frac{\omega_{0 s}}{\omega_{0}}\right)^{2}\right]^{2}\left(\frac{\partial \theta_{2}}{\partial I_{n 0}^{\prime}(k)}-\frac{\partial \theta_{1}}{\partial I_{n 0}^{\prime}(k)}\right) \\
& -\frac{2 L_{1}}{L_{0}}\left[1-\left(\frac{\omega_{0 s}}{\omega_{0}}\right)^{2}\right]^{2} \frac{x(k)}{I_{n 0}^{\prime \prime}(k)}-\frac{\pi R_{0}}{\omega_{0} L_{0} F_{s n}} \\
\frac{\partial F_{3}}{\partial V_{n g}(k)}= & b_{3}=-\frac{Z_{0}}{\omega_{0} L_{0}}\left[1-\left(\frac{\omega_{0 s}}{\omega_{0}}\right)^{2}\right]^{2} \frac{\pi}{F_{n s}^{2}}+\frac{\pi R_{0} I_{n 0}^{\prime}}{\omega_{0} L_{0} F_{n s}^{2}} \\
\frac{\partial F_{3}}{\partial V_{n g}(k)}= & c_{3}=-\frac{Z_{0}}{\omega_{0} L_{0}}\left[1-\left(\frac{\omega_{0 s}}{\omega_{0}}\right)^{2}\right]^{2}\left(\frac{\partial \theta_{2}}{\partial V_{n g}(k)}-\frac{\partial \theta_{1}}{\partial V_{n g}(k)}\right) .
\end{aligned}
$$

\section{Other Parameters}

Below are other parameters relevant for the derivation of the coefficients of (32) and (33).

1) Partial Derivatives for Paramter $V_{n m 2}$ :

$$
\begin{aligned}
\frac{\partial V_{n m 2}}{\partial y(k)} & =\frac{y(k)+I_{n 0}^{\prime}(k)}{V_{n m 2}} \\
\frac{\partial V_{n m 2}}{\partial x(k)} & =\frac{x(k)-1}{V_{n m 2}} \\
\frac{\partial V_{n m 2}}{\partial I_{n 0}^{\prime}(k)} & =\frac{y(k)+I_{n 0}^{\prime}(k)}{V_{n m 2}} \\
\frac{\partial V_{n m 2}}{\partial V_{n g}(k)} & =\frac{x(k)+1}{V_{n m 2}} .
\end{aligned}
$$

2) Partial Derivatives for Parameter $\theta_{1}$ :

$$
\begin{aligned}
\frac{\partial \theta_{1}}{\partial y(k)} & =\frac{\left.\left(\frac{x(k)}{I_{n 0}^{\prime}(k)}+I_{n 0}^{\prime}(k)\right) H x y(k)+I_{n 0}^{\prime}(k)\right)}{V_{n m 1}^{2} \sqrt{V_{n m 1}^{2}-\left(\frac{x(k)}{I_{n 0}^{\prime}(k)}+I_{n 0}^{\prime}(k)\right)^{2}}} \\
\frac{\partial \theta_{1}}{\partial x(k)} & =\frac{\left(\frac{x(k)}{I_{n 0}^{\prime}(k)}+I_{n 0}^{\prime}(k)\right)(x(k)+1) I_{n 0}^{\prime}(k)-V_{n m 1}^{2}}{V_{n m 1}^{2} I_{n 0}^{\prime}(k) \sqrt{V_{n m 1}^{2}-\left(\frac{x(k)}{I_{n 0}^{\prime}(k)}+I_{n 0}^{\prime}(k)\right)^{2}}} \\
\frac{\partial \theta_{1}}{\partial V_{n g}(k)} & =\frac{\left(\frac{x(k)}{I_{n 0}^{\prime}(k)}+I_{n 0}^{\prime}(k)\right)(x(k)+1)}{V_{n m 1}^{2} \sqrt{V_{n m 1}^{2}-\left(\frac{x(k)}{I_{n 0}^{\prime}(k)}+I_{n 0}^{\prime}(k)\right)^{2}}}
\end{aligned}
$$




$$
\begin{aligned}
\frac{\partial \theta_{1}}{\partial I_{n 0}^{\prime}(k)}= & \frac{\left(\frac{x(k)}{I_{n 0}^{\prime}(k)}+I_{n 0}^{\prime}(k)\right)\left[\left(y(k)+\frac{x^{2}(k)}{I_{n 0}^{3 \prime}(k)}\right)\right]}{V_{n m 1}^{2} \sqrt{V_{n m 1}^{2}-\left(\frac{x(k)}{I_{n 0}^{\prime}(k)}+I_{n 0}^{\prime}(k)\right)^{2}}} \\
& -\frac{\left(1-\frac{x(k)}{I_{n 0}^{2 \prime}(k)}\right)\left(V_{n m 1}^{2}-\left(\frac{x(k)}{I_{n 0}^{2 \prime}(k)}\right)\right)}{V_{n m 1}^{2} \sqrt{V_{n m 1}^{2}-\left(\frac{x(k)}{I_{n 0}^{\prime}(k)}+I_{n 0}^{\prime}(k)\right)^{2}}} .
\end{aligned}
$$

3) Partial Derivatives for Parameter $\theta_{2}$ :

$$
\begin{aligned}
\frac{\partial \theta_{2}}{\partial y(k)} & =\frac{x(k)+1}{V_{n m 1}^{2}} \\
\frac{\partial \theta_{2}}{\partial x(k)} & =\frac{-y(k)-I_{n 0}^{\prime}(k)}{V_{n m 1}^{2}} \\
\frac{\partial \theta_{2}}{\partial y(k)} & =\frac{x(k)+1}{V_{n m 1}^{2}} \\
\frac{\partial \theta_{2}}{\partial x(k)} & =\frac{-y(k)-I_{n 0}^{\prime}(k)}{V_{n m 1}^{2}} .
\end{aligned}
$$

4) Partial Derivatives for Parameter $\theta_{3}$ :

$$
\begin{aligned}
& \frac{\partial \theta_{3}}{\partial y(k)}=\frac{\left(\frac{x(k)}{I_{n 0}^{\prime}(k)}-I_{n 0}^{\prime}(k)\right)\left(y(k)+I_{n 0}^{\prime}(k)\right)}{V_{n m 2}^{2} \sqrt{V_{n m 1}^{2}-\left(\frac{x(k)}{I_{n 0}^{\prime}(k)}+I_{n 0}^{\prime}(k)\right)^{2}}} \\
& \frac{\partial \theta_{3}}{\partial x(k)}= \\
& \underline{\left(\frac{x(k)}{I_{n 0}^{\prime}(k)}-I_{n 0}^{\prime}(k)\right)(x(k)+1) I_{n 0}^{\prime}(k)-V_{n m 1}^{2}+2 x(k)+2 I_{n 0}^{2 \prime}(k)} \\
& V_{n m 2}^{2} I_{n 0}^{\prime}(k) \sqrt{V_{n m 1}^{2}-\left(\frac{x(k)}{I_{n 0}^{\prime}(k)}+I_{n 0}^{\prime}(k)\right)^{2}} \\
& \frac{\partial \theta_{3}}{\partial I_{n 0}^{\prime}(k)}=\frac{\left(\frac{x(k)}{I_{n 0}^{\prime \prime}(k)}+1\right)\left[V_{n m 1}^{2}-\left(\frac{x(k)}{I_{n 0}^{\prime}(k)}+I_{n 0}^{\prime}(k)\right)^{2}\right]}{V_{n m 2}^{2} \sqrt{V_{n m 1}^{2}-\left(\frac{x(k)}{I_{n 0}^{\prime}(k)}+I_{n 0}^{\prime}(k)\right)^{2}}} \\
& +\frac{\left(\frac{x(k)}{I_{n 0}^{\prime}(k)}-I_{n 0}^{\prime}(k)\right)\left(y(k)+\frac{x^{2}(k)}{I_{n 0}^{3 \prime}(k)}\right)}{V_{n m 2}^{2} \sqrt{V_{n m 1}^{2}-\left(\frac{x(k)}{I_{n 0}^{\prime}(k)}+I_{n 0}^{\prime}(k)\right)^{2}}} \\
& \frac{\partial \theta_{3}}{\partial V_{n g}(k)}=\frac{\left(\frac{x(k)}{I_{n 0}^{\prime}(k)}-I_{n 0}^{\prime}(k)\right)(x(k)+1)}{V_{n m 2}^{2} \sqrt{V_{n m 1}^{2}-\left(\frac{x(k)}{I_{n 0}^{\prime}(k)}+I_{n 0}^{\prime}(k)\right)^{2}}} .
\end{aligned}
$$

\section{REFERENCES}

[1] Lau, B. Y. (1988)

Small-signal input-to-output frequency response of switching converters.

In IEEE Power Electronics Specialists Conference Record, 2 (Apr. 1988), 1355-1363.

[2] Brown, A. R., and Middlebrook, R. D. (1981)

Sampled data modeling of switching regulators.

In IEEE Power Electronics Specialists Conference Record, 1981, 349-369.

[3] Redl, R., Molnar, B., and Sokal, N. O. (1984)

Small-signal dynamic analysis of regulated class-E $\mathrm{dc} / \mathrm{dc}$ converters.

In IEEE Power Electronics Specialists Conference Record, 1984, 62-71.

[4] Middlebrook, R. D., and Cuk, S. (1976)

A general unified approach to modeling switching converter power stages.

In IEEE Power Electronics Specialists Conference Record, 1976, 18-34.

[5] King, R. J., and Stuart, T. A. (1985)

Small-signal model for the series resonant converter. IEEE Transactions on Aerospace and Electronic Systems, AES-21, 3 (May 1985), 301-319.

[6] Panov, Y. V., and Lee, F. C. (1994)

A novel control evaluation technique for resonant converters.

In IEEE Power Electronics Specialists Conference Record, 1994, 301-308.

[7] Verghese, G. C., Elbuluk, M. E., and Kassakian, J. G. (1986) A general approach to sampled-data modeling for power electronic circuits.

IEEE Transactions on Power Electronics, PE-1 (Apr. 1986), 76-89.

[8] Vlatkovic, V., Sabate, J., Ridley, R., Lee, F. C., and Cho, B. (1992)

Small-signal analysis of the phase-shifted PWM converter. IEEE Transactions on Power Electronics, 7, 1 (Jan. 1992), 128-135.

[9] Vorperian, V., Tymerski, R., and Lee, F. C. (1985)

Equivalent circuit for resonant and PWM switches. IEEE Transactions on Power Electronics, PE-4, 2 (Apr. 1985), 205-214.

[10] Middlebrook, R. D., and Cuk, S. (1983)

Advances in Switched-Mode Power Conversion, Vols. 1 and 2.

Teslaco: Pasadena, CA, 1983.

[11] Sanders, S. R., Noworolski, J. M., Liu, X. Z., and Verghese, G. C. (1990)

Generalized averaging method for power conversion circuits.

In IEEE Power Electronics Specialists Conference Record, June 1990, 333-340.

Vorperian, V. (1990)

Simplified analysis of PWM converters using the model of the PWM switch: Parts I and II.

IEEE Transactions on Aerospace and Electronic Systems, 26, 3 (1990), 490-505.

[13] Vorperian, V., and Cuk, S. (1983)

Small signal analysis of resonant converters.

In IEEE Power Electronics Specialists Conference Record, Albuquerque, NM, June 6-9, 1983, 269-283.

[14] Witulski, A. R., and Erickson, R. W. (1987)

Small signal ac equivalent circuit modelling of the series resonant converter.

In IEEE Power Electronics Specialists Conference Record, 1987, 693-704. 
Small signal analysis of resonant converters and control approaches for parallel-connected converter systems. Ph.D. dissertation, University of Illinois at Chicago, Department of Electrical Engineering and Computer Science, Chicago, IL, July 24, 1991.

[16] Siri, K., Lee, C. Q., and Fang, S. J. (1990) Frequency response of resonant converters. In Proceedings of IEEE Industrial Electronics Conference, Pacific Grove, CA, Nov. 1990, Vol. II, 944-949.

[17] Elbuluk, M. E., Verghese, G. C., and Kassakian, J. G. (1988) Sampled-data modeling and digital control of resonant converters.

IEEE Transactions on Power Electronics, 3 (July 1988), 344-354.

[18] Vorperian, V. (1989)

Approximate small-signal analysis of the series and the parallel resonant converters.

IEEE Transactions on Power Electronics, 4 (Jan. 1989), $15-24$.

[19] Siri, K., Fang, S. J., and Lee, C. Q. (1991)

State-plane approach to frequency response of resonant converters.

IEE Proceedings, Pt.-G (Electronics Circuit and Systems), 138, 5 (Oct. 1991), 557-563.

[20] Witulski, A. R., and Erickson, R. W. (1987)

Small signal ac equivalent circuit modeling of the series resonant converter.

In IEEE Power Electronics Specialists Conference Record, 1987, 693-704.

[21] Batarseh, I., and Siri, K. (1993)

Generalized approach to the small signal modeling of dc-to-dc resonant converters.

IEEE Transactions on Aerospace and Electronic Systems, 29, 3 (July 1993), 894-909.

[22] Siri, K., Batarseh, I., and Lee, C. Q. (1993)

Frequency response for the conventional parallel resonant converter based on the state-plane diagram.

IEEE Transactions on Circuits and Systems, 40, 1 (Jan. 1993), 33-42.
[23] Yang, E. X., and Lee, F. C. (1992)

Small signal modeling of LLC-type series resonant converter.

HFPC'92, San Diego, CA, May 3-7, 1992, 186-197.

[24] Yang, E. X., Lee, F. C., and Jovanovic, M. (1992)

Small signal modeling of LCC-type parallel resonant converter.

In IEEE Power Electronics Specialists Conference Record, 1992, 941-948.

[25] Steigerwald, R. L. (1988)

A comparison of half-bridge resonant converter topologies.

IEEE Transactions on Power Electronics, 3 (Apr. 1988), 174-182.

[26] Bhat, A. K. S. (1993)

Analysis and design of a series-parallel resonant converter. IEEE Transactions on Power Electronics, 8, 1 (Jan. 1993), $1-11$.

[27] Batarseh, I., Liu, R., Lee, C. Q., and Upadhyay, A. K. (1990) Theoretical and experimental studies of the LCC-type parallel resonant converter.

IEEE Transactions on Power Electronics, 5, 2 (Apr. 1990), 140-150.

[28] Batarseh, I., and Lee, C. Q. (1989)

High frequency high order parallel resonant converter. IEEE Transactions on Power Electronics, 36, 4 (Nov. 1989), 485-495.

[29] Batarseh, I. (1990)

Analysis and design of high order parallel resonant converters.

Ph.D. dissertation, University of Illinois at Chicago, June 1990.

[30] Oruganti, R., and Lee, F. C. (1985)

Resonant power processing: Part I-state-plane analysis. IEEE Transactions on Industry Applications, (Nov. 1985), $1443-1460$

[31] Oruganti, R., and Lee, F. C. (1985)

Resonant power processing: Part II-method of control. IEEE Transactions on Industry Applications, (Nov. 1985), $1461-1471$ 

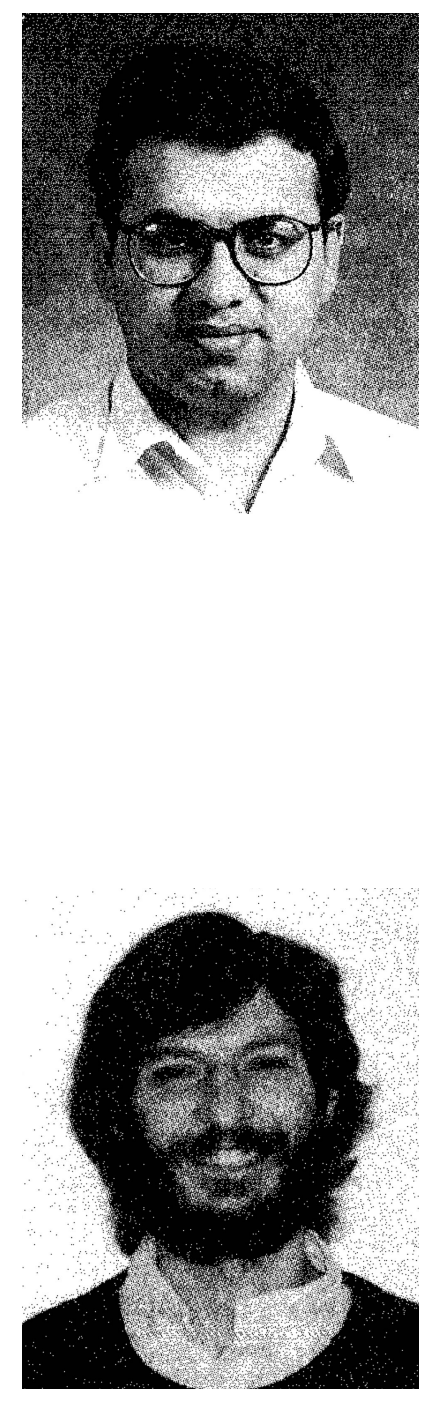

Issa Batarseh (S'83-M'91-SM'92) was born in Jordan on January 29, 1961. He received his B.S., M.S., and Ph.D. from the University of Illinois at Chicago in 1983, 1985, and 1990, respectively, all in electrical engineering.

He was a visiting Assistant Professor in the Electrical Engineering Department at Purdue University Calumet, from 1989 to 1990. In August 1991, he joined the Department of Electrical and Computer Engineering at the University of Central Florida in Orlando, as an Assistant Professor. He is currently engaged in research in the area of power electronics. His research interests include PWM and high frequency resonant converters, power factor correction circuits, and small signal modeling of dc-to-dc resonant converters.

Dr. Batarseh is a member of Tau Beta Pi and Eta Kappa Nu. Presently serving as Chairman of the IEEE-PE/IAS/PEL Orlando Chapter. From 1991-1993 he served as advisor to Eta Kappa $\mathrm{Nu}$ at the University of Central Florida. He is a registered Professional Engineer in Florida. He has served on the program committees of IEEE APEC, PESC, IECON and IAS.

Mario Sznaier (M'XX) received the Ingeniero Electronico and Ingeniero en Sistemas de Computacion degrees from the Universidad de la Republica, Uruguay in 1983 and 1984, respectively, and the MSEE and Ph.D. degrees from the University of Washington, Seattle, in 1986 and 1989, respectively.

He spent 1990 as a Research Fellow in Electrical Engineering at California Institute of Technology. From 1991 to 1993 he was an Assistant Professor of Electrical Engineering at the University of Central Florida. In 1993 he joined the Department of Electrical Engineering at Pennsylvania State University, where he is currently an Assistant Professor. His research interest include multiobjective robust control, $l^{1}$ and $\mathcal{H}_{\infty}$ control theory, applications of robust control to power electronics, and legged locomotion.

In 1992 Dr. Sznaier was awarded a National Science Foundation Research Initiation Award for his research on robust control of systems under mixed time/frequency domain performance specifications. He is a member of SIAM, Tau Beta Pi, and Eta Kappa Nu. From 1992 to 1993 he served as the faculty advisor to the IEEE Student Branch at UCF, and from 1993 he has been a co-advisor to Penn State's IEEE Student Branch. He served in the program committee of the 1994 IEEE CDC and is a member of the Control Systems Society Conference Editorial Board.

C. Magalemos photograph and biography not available. 